\title{
ASPECTOS QUIRURGICOS EN EL TRATAMIENTO DE LA TUBERCULOSIS GENITAL FEMENINA
}

\author{
Doctores Hernando Amaya León *, Alvaro Fonnegra Miramón**, \\ y Roberto Vergara Támara***
}

En el V Congreso Nacional de Obstricia y Ginecología (Medellín, 1963) (1) presentamos como ponencia un estudio bastante completo de la tuberculosis genital femenina en nuestro medio; de allí extractamos gran parte del material que hoy queremos analizar.

La intensificación de publicaciones científicas sobre la materia se inició en las primeras décadas de este siglo; en Colombia, tan sólo en 1950 se publicó, por uno de nosotros (2), un trabajo organizado aprovechando la revisión de dos hospitales bogotanos; de entonces acá se han escrito diversas monografías de interés $(3,4,5,6,7,8$, $9,10,11)$.

Quisiéramos advertir que, sin entrar en detalles, hasta 1950 los casos diagnosticados como TBC en Bogotá eran poquísimos; de 1950 a 1960 la casuística se elevó notablemente, y de 1960 a hoy estamos encontrando más y más casos de tuberculosis genital femenina; recientemente, Acero (12) en un trabajo sobre Endometritis TBC relieva que el $77 \%$ de los casos estudiados fueron hallados entre 1960 y 1963, habiendo revisado estadísticas desde 1954; esto indica con nitidez que se piensa más en la entidad y que los medios de diagnóstico han mejorado.

Para diagnosticar tuberculosis genital femenina es necesario pensar en ella; y éstos son los elementos de orientación clínica que nosotros insistimos como pilares básicos en tal sentido:

\section{CUADRO 1}

\section{ELEMENTOS DE ORIENTACION CLINICA}

Historia familiar de tuberculosis.

Antecedentes personales de tuberculosis..

Infertilidad.

Mal estado general crónico.

Masas anexiales en vírgenes.

Enfermedad pélvica inflamatoria crónica resistente a los tratamientos usuales.

* Jefe del Departamento de Obstetricia y Ginecología, Facultad de Medicina, Universidad Nacional. Bogotá, Colombia.

** Jefe de la Unidad de Ginecología, Facultad de Medicina, Universidad Nacional. Bogotá.

*** Jefe de la Unidad de Ginecología, Facultad de Medicina, Universidad Javeriana. Bogotá. 
Y así, cualquier enferma que presente uno o varios de estos elementos de sospecha debe ser investigada en detalle.

He aquí una síntesis de los métodos de diagnósticos empleados por nosotros:

\section{CUADRO 2}

\section{METODOS DIAGNOSTICO}

Biopsias de endometrio, cuello, etc. Raspado endometrial

Piezas quirúrgicas

Baciloscopia

Cultivos de sangre menstrual.

Cultivos de tejidos

Lavado endometrial.

Inoculación al curí

Tuberculina

Radiografía

Culdoscopia

Histerosalpingografía.

No es difícil presumir que cumpliendo con estas diferentes etapas de manejo consciente de nuestras pacientes sospechosas, es posible llegar a un diagnóstico positivo de tuberculosis genital femenina en cualquiera de sus localizaciones.

Y bien, ¿qué tratamiento aconsejamos? Debemos recordar primero la terapia médica.

Ante todo, y como es de rigor, al establecer la positividad del cultivo por el procedimiento que se sigue en el laboratorio de TBC del Instituto Nacional de Salud, se obtiene a la vez el antibiograma; ésto es obvio, nos previene contra las resistencias; paso siguiente, la búsqueda de la concentración con- siderada óptima entre nosotros, de las drogas tuberculostáticas en los niveles sanguíneos: 0.4 microgramos de isoniacida por cc. de suero y 20-25 microgramos de estreptomicina en el misvo volumen sanguíneo, dejando el PAS únicamente como potencializador de !a isoniacida; estos niveles deben ser revisados con alguna frecuencia.

De acuerdo con el Hospital Santa Clara, hemos empleado el siguiente esquema, que presentamos al IV Congreso Mundial de Obstetricia y Ginecología (Buenos Aires, 1964) (13) y que viene dando muy buenos resultados: 1 gr. diario de estreptomicina y 16 miligramos de isoniacida por kilo de peso, durante 3 meses, agregando piridoxina; luego se reduce la estreptomicina a 2 gramos semanales y se mantienen las dos drogas por un mínimo de un año. Si por resistencia o intolerancia a estos fármacos se necesita el PAS, la dosis de 6 gramos diarios es adecuada. En casos de resistencia a todas estas drogas, empleamos cicloserina en dosis de 500 miligramos diarios o la etionamida a razón de 15 mg. por kilo de peso y por día, con la consigna de vigilar muy de cerca sus severas complicaciones.

No podemos dejar da consignar el hecho importante de que los corticosteroides son arma eficaz complementaria, y en especial cuando el B. de Koch afecta mucosas; ésto adquiere valor especial en las localizaciones tubáricas, administrados en forma sistémica o localmente.

Cuando se ha resuelto intervenir quirúrgicamente un caso de tuberculosis genital femenina, es imperativo 
prepararlo con terapia médica por lo menos durante 3 o 4 meses, y continuar luego el tratamiento hasta completar por lo menos 1 año.

Con estas consideraciones, entramos ahora a determinar la terapia quirúrgi$c a$ en la tuberculosis genital femenina.

Iniciamos este aspecto del tema presentando nuestras últimas revisiones estadísticas:

CUADRO 3

INFECCIONES E INFLAMACIONES PELVICAS

Historias revisadas (Nueve años de revisión, marzo 1956 a marzo 1965) . . . . . . . . . 765

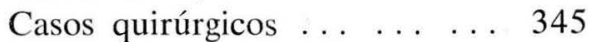

Con anatomía pato'ógica ... $328^{1}$

Casos con diagnóstico positivo para T.B.C. ... ... ... ... Casos de T.B.C. previamente diagnosticados ..........

Casos insospechados .......

$195 \%$.

$220 \%$.

El primer comentario se orienta a resaltar el hecho, para nosotros muy valioso, de que analizando el material empleado en un estudio investigativo sobre enfermedad pélvica inflamatoria en el Hospital San Juan de Dios, encontramos un $20 \%$ de etiología tuberculosa demostrada a la histología, localizada en trompas, sitio e!ectivo de la enfermedad en el casi $100 \%$ de los casos; y ésto nos da un índice muy importante de que la incidencia baja hasta ahora relatada en nuestros estudios no parece real sino producto de investigación previa insuficiente de la en- tidad y de no haber llevado una metodología adecuada en su búsqueda.

CUADRO 4

T.B.C. PELVI-PERITONEAL

Casos diagnosticados de T.B.C.

$1956-1963 \ldots \ldots 20 \%$

Casos diagnosticados de T.B.C.

$1963-1965 \ldots \ldots \ldots$.... $40 \%$

Pero surge de inmediato el comentario al cuadro 4, de que en la etapa hasta 1960 los casos revisados llegaron en su gran mayoría a la mesa operatoria sin diagnóstico previo acertado y sin tratamiento médico lógico; de entonces acá, esas cifras cambian, sufriendo una evolución favorable rápida. El personal de nuestros servicios hospita!arios universitarios ya ha adquirido la mentalidad de sospechar ta tuberculosis genital femenina, y en San Juan de Dios viene operando una clínica dedicada a esta labor, dirigida por el jefe de la unidad Ginecológica del Departamento.

Por los motivos expuestos, antes no teníamos un criterio definido en frente al caso de TBC genital, que casi siempre era hallazgo operatorio casual; en la actualidad, fruto de nuestra experiencia y aprovechando la de estadísticas foráneas, hemos l'egado a formalizar pautas que ahora informamos.

Recordemos que entre las tendencias de extrema, o sean, la de la escuela inglesa con Stalworty $(14,15)$ y Sutherland (16) a la cabeza quienes sostienen que la cirugía debería casi proscribirse en el tratamiento de la tuber- 
culosis genital femenina, y la de la vienesa de Knaus (17)) quien afirma que la cirugía es el único tratamiento curativo, hay una tendencia casi universal en el resto de las escuelas a considerar la terapia mixta como ideal, y con indicaciones más o menos precisas. Sin embargo, hasta el momento nadie ha podido hacer una sistematización tentativa del problema.

Revisemos algunas estadísticas recientes:

Szolga (18), de Budapest, opera 50 de 80 pacientes; $60 \%$ más o menos.

Kardos (19), también de Budapest, consigna $36 \%$ entre 557 pacientes tratadas por esta enfermedad.

Kräubig (20), relata una incidencia de $25 \%$ de pacientes intervenidas en su clínica (Alemania) y afirma además de que un $75 \%$ tratadas médicamente, permanecerían curadas luego de 5 años de control.

Poland (21), relata $57 \%$ de intervenciones en 140 casos, de los cuales 17 fueron sometidos a histerectomía con salpingectomía bilateral, o sea el $12 \%$, ignorándose el tipo de cirugía en el resto y el número de casos intervenidos con diagnóstico previo.

Henderson y col. (22), de Toronto, Canadá, sostienen que con tratamiento médico adecuado, menos del $20 \%$ requieren cirugía.

Nuestras propias estadísticas no tienen hasta el momento valor definido, pues darían una muy alta incidencia operatoria, debida en parte al gran porcentaje de casos insospechados, como se anotó previamente; pero en la actualidad, ya hemos reportado resultados con la terapia médica que podemos considerar altamente satisfactorios; y estamos convencidos de que con buen diagnóstico y una terapéutica tuberculostática adecuada, los casos quirúrgicos se hacen cada vez menos indispensables.

He aquí las pautas que en la actualidad preconizamos en nuestros servicios hospitalarios:

\section{CUADRO 5}

Consideraciones importantes para ESCOGER TERAPEUTICA

\section{Forma clínica:}

Leve: Unicamente la serosa está atacada (miliar).

Moderada: El órgano está incluído, pero libre (exudativa).

Grave: Varios órganos enfermos y con adherencias íntimas (incluyendo vejiga, intestino, mesenterio, etc.) (fibro-caseosa, productivo adhesiva).

Edad: 20 a 30 años

30 a 40 años

40 o más.

Esterilidad: Primaria.

Secundaria.

Gestaciones previas. 


$$
\text { CUADRO } 6
$$

\section{CONSIDERACIONES TERAPEUTICAS EN MUJERES JOVENES}

$\begin{array}{llll}\text { Edad } & 20-30 & 20-30 & 20-30 \\ \text { Forma clínica } & \text { Leve } & \text { Moderada } & \text { Grave } \\ \text { Esterilidad } & \text { Sí } & \text { Sí } & \text { Sí } \\ \text { Terapia médica: } & \searrow & \searrow & \searrow \\ \text { Terapia quirúrgica: } & \text { Estrictamente } & \text { Unica, o pre y } & \text { Preoperatoria 4 } \\ & \quad \text { Ocasionalmen- } & \text { postoperatoria } & \text { meses y postope- } \\ & \text { te reparadora } & \text { Según controles } & \text { ratoria por 1 año } \\ & & \text { histológicos y } & \text { Según invasión de } \\ & & \text { bacteriológicos. } & \text { lesiones. Tenden- } \\ & & \text { Tendencia con- } & \text { cia conservadora } \\ & & \text { servadora. } & \text { (?). }\end{array}$

\section{CUADRO 7}

CONSIDERACIONES TERAPEUTICAS EN MUJERES MADURAS

$\begin{array}{ll}\text { Edad } & 30-4 \\ \text { Forma clínica } & \text { Leve } \\ \text { Esterilidad } & \text { Si o ? } \\ & \downarrow \\ \text { Terapia médica } & \text { Unica } \\ \text { Terapia } & \downarrow \\ \text { quirúrgica } & \text { No }\end{array}$

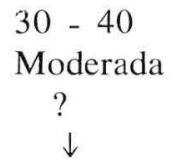

Moderada

?

$\downarrow$

Pre y postoperatoria<smiles>[AlH2]</smiles>

Tendencia:

Conservadora: hacia los 30

Radical: hacia los

40 .
$30-40$

Grave

$\stackrel{?}{\downarrow}$

Pre y postoperatoria $\downarrow$

Tendencia:

Radical.

\section{CUADRO 8}

Conducta en casos insospechados OPERATORIOS

Pacientes jóvenes y lesiones:

Leves: Cerrar y tratamiento médico. Moderadas: Cirugía muy conservadora y tratamiento médico intensivo. Graves: Generalmente cerrar y tratamiento médico intensivo; luego: reintervenir. Criterio conservador (?) y continuar tratamiento médico.
Pacientes maduras y lesiones:

Leves: Cerrar y tratamiento médico. Moderadas: Cirugía con tendencia amplia y tratamiento médico postoperatorio intensivo.

Graves: Según caso clínico: mucho cuidado por complicaciones.

a) Cerrar, tratamiento médico y operación radical.

b) Operación radical y tratamiento intensivo posterior. 
Es obvio que estas consideraciones no pueden tomarse como normas rígidas; cada caso debe ser analizado en particular, y lo importante, es conocer a fondo el problema. Sirven eso sí, de orientación didáctica y de aplicación práctica en la terapia quirúrgica de la tuberculosis genital femenina, en especial cuando el cirujano ginecólogo debe resolver un caso cuya previsión no pueda estar orientada por una experiencia amplia y suficiente.

\section{Resumen:}

1. Después de estudiar trabajos nacionales y extranjeros, no hay pautas suficientes para el manejo quirúrgico adecuado de las pacientes con tuberculosis genital femenina.

2. Los autores, pertenecientes a dos escuelas de Medicina de la ciudad de
Bogotá, consideran que es posible curar un buen número de estas enfermas con tratamiento médico bien llevado y orientado; sus experiencias a este respecto son satisfactorias.

3. Se insiste en la necesidad de pensar en la tuberculosis para llegar a sospecharla y se recuerdan elementos de orientación clínica y metodología de diagnóstico.

4. Se ponen a consideración cuadros de orientación terapéutica, tenien. do en cuenta la edad de la enferma, forma clínica de la entidad, esterilidad, etc.

5. Estos cuadros no pueden ser considerados como normas rígidas sino como elementos didácticos y de orientación terapéutica.

\section{BIBLIOGRAFIA}

1. AMAYA LEON H. y col.: Tuberculosis genital femenina. Estudio clínico, diagnóstico y estadístico en Bogotá (Colombia). Rev. Col. de Obst. y Ginec., XV: 1964.

2. AMAYA LEON H.: Tuberculosis en Ginecología. Rev. Col. de Obst. y Ginec., I: 227, 1950.

3. AMAYA LEON H.: Hidrazida del ácido isonicotínico en el tratamiento de enfermas tuberculosas embarazadas. Rev. Col. Obst. y Ginec., $\mathrm{V}:$ 5, 1953.

4. VAlenCiA P. G.: Tuberculosis Genital en la mujer. Rev. Col. de Obst. y Ginec., VII: 69, 1955.

5. AMAYA LEON H., CAMERO R., GOMEZ C.: Endomstrio Tuberculoso. Memorias de la II Convención Colombiana de Obst. y Ginec. Cali, 1955.

6. CRUZ A: Dos casos de Piosalpinix tuberculoso. Rev. Col. de Obst. y Ginec., XI: 597, 1960.

7. RODRIGUEZ G. H.: Tuberculosis genital. Diagnóstico. Rev. Col. de Obst. y Ginec., X: 152, 1959. 
8. CHAPARRO M. J.: Tuberculosis genital en el Hospital de la Samaritana. Tesis de grado, Universidad Javeriana, 1960.

9. BOTERO J., RESTREPO S. J.: Tuberculosis endometrial y embarazo. Rev. Col. de Obst. y Ginec., XIII, 211, 1961.

10. BOTERO PELAEZ B., MONTES J., ACEBEDO F. O., GIRALDO A.: Tuberculosis genital femenina. Rev. Col. de Obst. y Ginec., XV: 137, 1954.

11. DURAN F., ARENAS I., REY P.: Tuberculosis genital. Rev. Col. de Obst. y Ginec. XV: 145, 1964.

12. ACERO U.: Endometritis Tuberculosa. Inédito.

13. AMAYA LEON H., VERGARA R., VILLARREAL J.: Tuberculosis genital femenina. Esquema de terapia médica. IV Congreso Mundial de Ginec. y Obst. Resumen de las Comunicaciones, pág.. 6, Ed. Américas, 1964.

14. STALLWORTHY J.: Genital tuberculosis in the female. J. Obst. Gynec. Brit. Emp., 61: 614, 1952.

15. STALLWORTHY J.: Fertility and genital tuberculosis. Fert. and Sterol., 3: $284,1963$.

16. SUTHERLAND A. M.: Genital tuberculosis in women. Am. J. Obst. Gynec., 79: 486, 1960.

17. KNAUS H. H.: Surgical treatment of genital and peritoneal tuberculosis in the female. Am. J. Obst. Gynec., 83: 73, 1962.

18. SZOLGA I.: Peritonitis tuberculosa y tuberculosis genital en la mujer. Zentralbl. Gynäk.s 85: 580, 1963.

19. KARDOS F.: Experiencias quirúrgicas con tuberculosis genital femenina. Zentralbl. Gynäk., 85: 584, 1963.

20. KRAUBIG H.: Cit. Year Book of Gynec., 425, 1964-1965.

21. POLAND B. J.: Female pelvis tuberculosis with special reference to interfility. Am. J. Obst. Gynec., 91: 350, 1965.

22. HENDERSON N., HERKINS J. L., STITT J. F.: Pelvic tuberculosis. Am. J. Obst. Gynec., 80: 21, 1960. 